\title{
DETECTION OF SYNCHROTRON EMISSION FROM A UNIQUE HERBIG HARO OBJECT IN ORION
}

\author{
F. YUSEF-ZADEH \\ Dept. of Physics and Astronomy, Northwestern University \\ Evanston, IL 60201 \\ T. CORNWELL \\ NRAO, P.O. Box O, Socorro, NM 87801
}

B. REIPURTH AND M. ROTH

ESO, Karl-Schwarzschild-Strasse 2, D-8046

Garching bei München

\begin{abstract}
We have obtained radio continuum observations of an $\mathrm{HH}$ object, known as the "streamer" in Orion using the VLA in a number of configurations at $\lambda 2,6$ and $20 \mathrm{~cm}$. This object is located several arc-minutes to the north of HH34 in L1641 cloud and exhibits a remarkably long and narrow structure with dimensions of $5^{\prime} \times 10^{\prime \prime}$ in optical photographs unlike the morphology of any other $\mathrm{HH}$ object. Based on radio continuum observations, we have detected a significant linearly polarized emission at $\lambda 6 \mathrm{~cm}$ from the core of the streamer indicating highly uniform magnetic field geometry. This unusual result suggests that the source responsible for producing the nebulosity is a nonthermal-emitting radio source. Because of the presence of a number of newly born low-mass stars in L1641 and because of the detection of a highly reddened $2 \mu \mathrm{m}$ source coincident with the synchrotron source, it is likely that the source at the core of the streamer is a low-mass star responsible for the non-thermal emission and the $\mathrm{HH}$ nebulosity. Presence of synchrotron emission associated with a newly born star supports further view that the magnetic field plays a strong role in the early phase of star formation.
\end{abstract}

Detailed structure of the V571 "streamer" which lies a few arc-minutes north of the spectacular HH-34 in Orion and adjacent to T-Tauri star, V571, as shown in Fig. 1, was studied in detail by Reipurth and Sandell (1985, Astr.Ap., 150, 307). In order to identify the source responsible for producing such an unusual streamer structure which shows to have $\mathrm{HH}$ spectrum at its core, radio continuum and near-IR observations of this source were carried out using the VLA in its compact configurations and the $4 \mathrm{~m}$ telscope at CTIO.

Figure 2 shows the continuum map of the streamer at $\lambda 6 \mathrm{~cm}$ with a spatial resolution of $\sim 12^{\prime \prime} \times 12^{\prime \prime}$. The streamer is seen to consist of a discrete compact radio source from which elongated nebulosity appears to arise as it extends for about $2^{\prime}$ due southwest. The compact source coincides with the core of the optical streamer from which a number of arc-like structures appear to emanate (Reipurth and Sandell 1985). The extended structure with surface brightness of $\sim 0.1-0.2 \mathrm{mJy}$ is considered to be the radio continuum counterpart to the elongated $5^{\prime}$ structure seen as arc $A$ in 
the optical image of this source. This result is consistent with the lack of reflected emission from the streamer as reported by $\mathrm{RS}$. The J, H, and $\mathrm{K}$ observations indicate a weak 17 th magnitude near-IR source, in the core position. The position of the near-IR source coincides with the peak radio component.

We detected significant degree of linear polarization, 6-7\%, at the position of the compact source at $\lambda 6 \mathrm{~cm}$. The line segments which represent the degree of linear polarization are superimposed on the total intensity map of the core of the streamer at $\lambda 6 \mathrm{~cm}$ and shown in Figure 3 having a spatial resolution of $4.78^{\prime \prime} \times 3.4^{\prime \prime}$. We note the elongated morphology of the total and polarized intensity distributions is directed along the major axis of the extented optical nebulosity. The highest degree of polarization appears to arise from the central region of the discrete source surrounded by a halo of depolarized emission. A steep value of $\alpha \sim-1.2$ is also found when the comparison was made between the $\lambda 20$ and $6 \mathrm{~cm}$ maps with a spatial resolution of $18.3^{\prime \prime} \times 11.1^{\prime \prime}$. The $\lambda 20 \mathrm{~cm}$ peak flux of $16.9 \mathrm{mJy} /$ beam is a few times higher than that seen toward HH-1 and HH-2 in Orion. No significant polarized emission was detected at $\lambda 20 \mathrm{~cm}$.

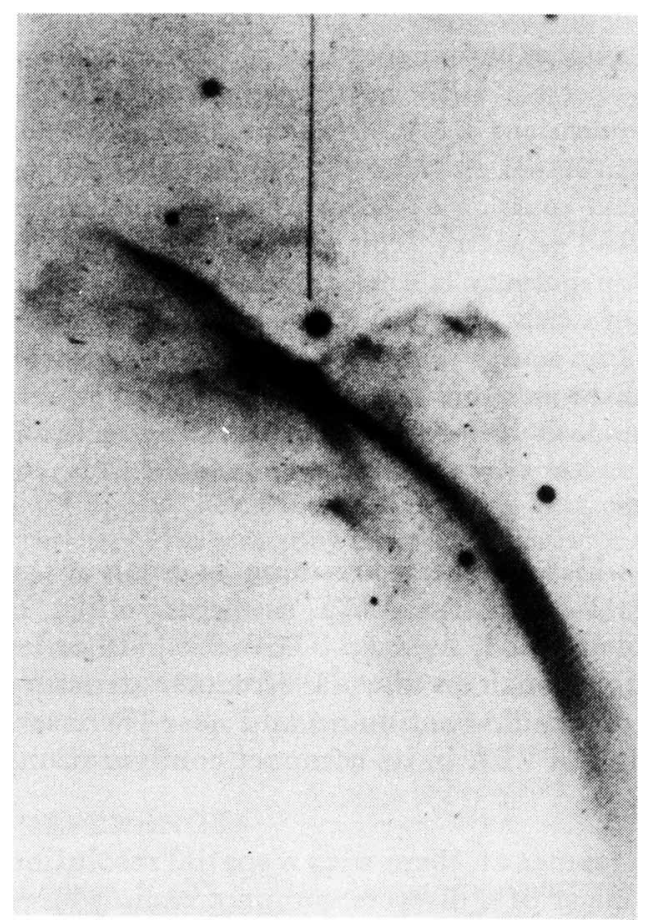

Fig. 1

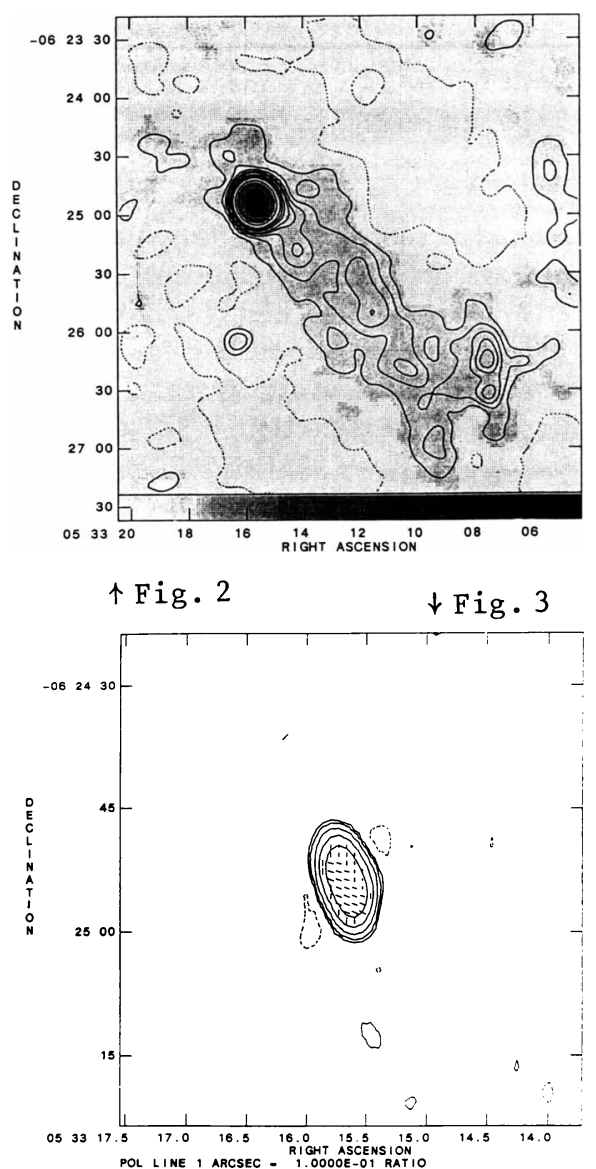

